\title{
Hacia Una Reconceptualización Teórica Y Metodológica Del Gobierno Abierto: Paradigma Explicativo De La Gobernabilidad Local
}

\author{
Lic. Alberto Ibarra Cortés \\ Pasante de la Maestría en administración con énfasis en Gestión pública del \\ Centro de investigación y estudios de posgrado \\ Universidad autónoma de San Luis Potosí - México \\ Dr. Louis Valentin Mballa \\ Dr. Mario Eduardo Ibarra Cortés \\ Dr. Miguel Ángel Vega Campos \\ Profesor Investigador del Centro de investigación y estudios de posgrado \\ Universidad autónoma de San Luis Potosí - México
}

\section{Resumen}

El objetivo de este artículo es realizar un acercamiento teórico y metodológico sobre la implementación del gobierno abierto en la gobernanza local. En realidad, el gobierno abierto surge por la falta de capacidad y recursos del Estado, donde la ciudadanía cada vez es más exigente e informada. Asimismo, el gobierno abierto busca que sean atendidos los problemas públicos inscritos en las agendas de los gobiernos locales; esto a través de la promoción de una cultura de trasparencia que facilite el acceso a la información con fines de control social, político y económico. A través de un método de revisión de literatura y diseño metodológico cualitativo, este artículo destaca que los cuatro principios del gobierno abierto son: la innovación, la transparencia, la participación ciudadana y la rendición de cuentas. El cumplimiento de la gobernanza con estos principios sin lugar a duda es garante de éxito en los espacios de gobernanza local.

Palabras Clave: Gobierno abierto, Problemas Públicos, Transparencia, Participación Ciudadana, Rendición de cuentas 


\title{
Towards a Theoretical and Methodological Reconceptation of Open Government: Explanatory Paradigm of Local Governance
}

\author{
Lic. Alberto Ibarra Cortés \\ Pasante de la Maestría en administración con énfasis en Gestión pública del \\ Centro de investigación y estudios de posgrado \\ Universidad autónoma de San Luis Potosí - México \\ Dr. Louis Valentin Mballa \\ Dr. Mario Eduardo Ibarra Cortés \\ Dr. Miguel Ángel Vega Campos \\ Profesor Investigador del Centro de investigación y estudios de posgrado \\ Universidad autónoma de San Luis Potosí - México
}

\begin{abstract}
The objective of this article is to carry out a theoretical and methodological approach on the implementation of open government in local governance. Open government arises because of the lack of capacity and resources of the State, where citizens are increasingly demanding and informed. Likewise, open government seeks to address the public problems inscribed in the agendas of local governments; this through the promotion of a culture of transparency that facilitates access to information for the purposes of social, political and economic control. Through a literature review method and qualitative methodological design, this article highlights that the four principles of open government are: innovation, transparency, citizen participation and accountability. The fulfillment of the governance with these principles without a doubt, is guarantor of success in local governance spaces.
\end{abstract}

Keywords: Open government, Public issues, Transparency, Citizen participation, Accountability

\section{Introducción}

Mucho se ha hablado respecto al tema del gobierno abierto en los últimos años; a través de este artículo, hacemos un acercamiento metodológico para la implementación de éste. El principio del gobierno abierto surge por la falta de recursos y capacidad del estado, de una ciudadanía que cada vez es más exigente e informada las cuales quieren tomar partido en las decisiones y 
acciones donde los gobiernos se preguntan cómo incorporar el gobierno abierto en sus capacidades organizacionales. Del mismo modo, el concepto de organización ha evolucionado en el tiempo lo cual ha permitido interpretar la sociedad que se organiza para alcanzar un objetivo; es por tal motivo debemos comprender que el gobierno abierto es un conjunto de organizaciones en interacción compleja.

En realidad, en la gobernabilidad, han incrementado la complejidad y la incertidumbre en la gestión de las organizaciones públicas debido a la descentralización, la pluralidad, la alternancia política y la existencia de una sociedad conflictiva; por lo que el principal reto de los gobiernos estatales es crear condiciones institucionales para la gobernabilidad. Para ello, los actror3es de toda índole necesitan de nuevos mecanismos que regulen su funcionamiento mediante la gobernabilidad entendida "capacidad de ejercer el poder en una estructura más participativa, diferente al modelo jerárquico" (Mballa, 2018). Para implementar un gobierno, las autoridades políticas deben ser fuertes y legítimamente democráticas para que, en su quehacer público, se van reflejados los intereses de todos los grupos sociales. Para que haya gobernabilidad los actores involucrados tienen que ser eficientes en su área de acción sin que ninguno domine a los de más y cooperar en el diseño, formulación e implementación de políticas públicas en un ambiente armonioso porque entran en juego, diferentes tipos de intereses.

El modelo de gestión del gobierno abierto plantea la necesidad de cambiar a los gobiernos estatales superando la estrategia administrativa que ha predominado, la clave está en poner como prioridad el cambiar mecanismos que determinan la estructura y funcionamiento de la administración pública. $\mathrm{La}$ tarea es encontrar las reglas y normas que permitan la pluralidad, transparencia, eficiencia y rendición de cuentas; para ello, debe establecer y garantizar el conjunto de normas en que se apoya la acción colectiva. En las últimas décadas en México ha sido testigo de un cambio en las formas de organización y acción gubernamental formado por un conjunto de valores, principios y técnicas llamadas ciencias de gestión. Asimismo, el gobierno en este marco hace referencia a dos ejes principales: la promoción de una cultura de transparencia institucionalizada y el fortalecimiento de los espacios de participación ciudadana.

Para elaborar este artículo, nos hemos apoyado en un método de revisión de literatura desde una perspectiva analítica. Este método nos permitido estipular que la generación de espacios de colaboración e innovación entre los diversos actores, particularmente entre las administraciones públicas, la sociedad civil y el sector privado, para codiseñar y/o coproducir valor público, social y cívico es la clave para la implementación del gobierno abierto. Asimismo, llegamos a la conclusión de que los nuevos roles del gobierno abierto han generados nuevos procesos de 
gobernanza y han modificado su estructura, modernizado sus procesos de trabajo y complejizado las alternativas de toma de decisiones. Estos nuevos procesos de gobierno pueden interpretarse como un síntoma de un fenómeno social más amplio, debido a los cambios no solo en lo económico, sino también en lo político y en la persistencia de los déficits sociales como la pobreza, la marginación y la exclusión social, en este contexto el gobierno abierto recupera espacios en la gestión de los asuntos públicos.

\section{1) Los principios del Gobierno Abierto. Una visión conceptual}

Una evolución que tal vez surgió de la capacidad y recursos limitados del Estado, de una ciudadanía cada vez más informada, exigente y con necesidades cambiantes que buscan sean atendidas o de la suma de las anteriores. El hecho es que el llamado a la apertura de los gobiernos es inminente, las personas quieren tomar partido en las decisiones y acciones que las involucran y se requiere canalizar esa intención hacia la construcción y los gobiernos han comenzado a preguntarse como incorporar el Gobierno Abierto en sus capacidades organizacionales (Naser y Ramírez: 2014).

\subsection{La visión organizacional, para la comprensión de la acción pública local}

A partir de las características más relevantes del concepto de organización se pueden mencionar los siguientes puntos que permiten comprender las acciones implantadas por el gobierno local: el concepto organización es definido primero en un sentido dinámico, ya que se refiere a una actividad que referencia a una dimensión de reconfiguración del todo social. La metáfora orgánica se refería a la funcionalidad del sistema, pero también resaltaba la dependencia jerárquica de sus partes frente a la dirección. El concepto moderno identificaba a la organización con el orden social, concepto que es reemplazado, más tarde, por la metáfora de la máquina, comprendido en un horizonte de racionalidad; este nuevo orden social fue considerado como un instrumento para el logro de objetivos organizacionales.

El concepto organización como mecanismo para la comprensión de la acción gubernamental local, es posible mediante un ajuste mutuo entre sus partes y de éstas al conjunto. En lugar del control exclusivo por parte de los primeros niveles jerárquicos del cuerpo social se considera que las partes del mecanismo - organismo social son capaces de autocontrol racional. El concepto se aplicaba al todo social, en el que el resultado de actividad es la estructuración racional de la dimensión organizada del Estado, es una concepción tecnocrática de la vida social.

La legitimidad del ordenamiento racional se deriva de la misma sociedad, la cual es concebida como un colectivo de individuos capaces de ordenar por sí mismos su convivencia con la finalidad de alcanzar objetivos conjuntos. La estructuración del mecanismo social que resulta de la 
organización no se basa en relaciones personalizadas, sino en relaciones definidas de forma abstracta tal como es planteado por el modelo burocrático. La regulación mutua de las partes tiene como objetivo la cooperación. La asignación de funciones es sólo un medio, la organización no tiene como finalidad la creación de niveles jerárquicos, sino establecer las condiciones para la cooperación de los individuos.

En síntesis, el concepto de organización ha sido una variable explicativa que evolucionó en el tiempo, lo cual lo ha convertido en un medio que permite interpretar la sociedad que se organiza para alcanzar un objetivo, por tal motivo es útil para comprender la acción gubernamental.

\section{2) El Gobierno Abierto como conjunto de organizaciones en interacción compleja}

Se conceptualiza al sistema por su diferencia al entorno, por ello el sistema desarrolla un grado de complejidad diferente al del entorno. Es por eso por lo que, su primera categoría analítica es la complejidad. El concepto es entendido como relacional, indica siempre una referencia a la capacidad de observación y análisis del observador. Hay complejidad cuando existen más relaciones que las observables para un observador con capacidad limitada (Luhmann: 1994). La complejidad es entendida dentro de su concepción del constructivismo social, en que el observador construye - observa un horizonte. Cuando la observación percibe y construye un número tal de relaciones que no puede abarcar o comprender en sus propios esquemas de interpretación, es decir, cuando percibe que no percibe todo, entonces considera "complejo" lo observado. Se trata de una complejidad en grado siempre relativo al observador. Lo que para el principiante es todavía complejo, puede dejar de serlo para el experto que domina más categorías o esquemas de explicación y percepción de nuevas realidades.

La relación con el entorno comprende una relación general, incierta y variable, por eso el polo que genera relaciones selectivas al entorno, más que relacionarse a un conjunto de entidades externas, se remite a los efectos que producen en él dichas relaciones, ya sea como efectos causados, informaciones o percepciones de algo improbable. La relación afirmada es la de complejidad reducida. La complejidad (Ibarra y Santos: 2011) refleja a nivel de marco teórico la experiencia cotidiana de lo inabarcable del entorno en el que funciona el sistema organizado: cultural, socio-político, económico y tecnológico, con sus innumerables implicaciones mutuas, que es lo que ha obligado a una división de funciones a nivel social, organizacional y especializadas en campos parciales de problemas del entorno general, que para el caso de las organizaciones públicas sería el desarrollo económico, educativo, salud, medio ambiente, servicios públicos, seguridad, entre otros más (Mballa: 2017). 
La creación de fronteras del sistema se da de manera dinámica como un proceso de transformación de mayor a menor complejidad y relacionalmente construido, en cuanto que afecta las relaciones internas o externas entre acciones y se realiza por selección de objetivos. El sistema sólo considera determinados segmentos del entorno que son relevantes y sólo se ocupa de determinados problemas o necesidades de dicho entorno. Un sistema organizado tiene que ser selectivo. El sistema autodefine sus límites, seleccionando sus tipos y cantidades de relaciones con el entorno. Esta reducción en su configuración supone una forma de afrontar la complejidad del entorno que se interioriza como elemento esencial en el funcionamiento del sistema.

Al trazar sus fronteras con el entorno, el sistema organizado crea su propia lógica de acción y define lo que para él es entorno relevante y lo que no lo es, así como sus posibles grados de relevancia en los segmentos del entorno que el mismo sistema proyecta. Esta reducción selectiva tiene un doble efecto: negativo y positivo. Se restringe el ámbito del planteamiento de problemas a resolver por el sistema, pero al mismo tiempo se crea una nueva dificultad, la selección implica indeterminación, incertidumbre y riesgo, pues se dejan fuera muchas áreas del entorno que no son analizadas y de las que se desconocerá totalmente su influjo e impacto en el propio sistema.

\section{3) El Gobierno Abierto desde el paradigma sistémico}

El paradigma de interpretación al pasar de uno que ponía énfasis en la relación causal por un enfoque sistémico orientado al tratamiento de la complejidad. Esto para March/Olsen (1987) implicaba una dificultad al decidir, al restringir la capacidad de conocer. La complejidad interna se manifiesta de forma concreta en las relaciones entre decisiones de las organizaciones que conforman al gobierno local. La decisión permite integrar historia previa de las organizaciones del gobierno estatal, experiencias anteriores, evaluaciones de influencias y poder. Al respecto Pettigrew (1973) establecía que una: "Teoría adecuada de la toma organizativa de decisiones deberá asumir el hecho de que las decisiones no son tomadas por los individuos o ocupantes de un rol, sino a través de procesos que están afectados por las características de la unidad o unidades en que se debe tomar la decisión".

Esto significa que en los gobiernos locales en lugar de la visión individualista hay que observar la realidad organizacional en referencia a las orientaciones o sentido propios de un colectivo, donde la racionalidad no es ya la de la adecuación de los fines - medios en la perspectiva o modo de observar individual, sino la definida por el horizonte común de sentido del conjunto de organizaciones del gobierno local como sistema organizado. Es una racionalidad sistémica, en la que se da la configuración de racionalidades 
parciales del tipo fin - medios, pero lo que no puede reducirse es el concepto de sistema al de un mero medio para la consecución de los fines.

Con este planteamiento se introduce una nueva diferencia, la del sentido del sistema y la del sentido de la acción individual de los miembros del gobierno local. Para otros, el sentido es crear valor en forma de productos o servicios destinados a satisfacer las necesidades de los usuarios de la organización. La diferencia está en la orientación del sujeto individual en que es primordial el objetivo de obtener el máximo de beneficios, o en la orientación del sistema considerado en cuanto sistema organizado. En la relación gobierno local y su entorno, el sistema organizado estatal tiene la característica de mantener una relación permanente y conflictiva. Éste actúa como un imperativo en la determinación de la agenda pública local y no sólo como una perturbación informativa para sus decisiones. Los problemas públicos del entorno pasan a problemas internos de gobierno cuando el gobierno local los asume en su agenda de acción. El proceso de apropiación se realiza mediante un proceso de decisión que establece una jerarquía de prioridades de acción para la resolución de situaciones socialmente críticas. El gobierno local, por su naturaleza política, está obligado a procesar las perturbaciones de su entorno y las tratará según sus propios esquemas de interpretación.

De esta manera se establece una dependencia lógica entre fines y medios, como vieron Cohen, March y Olsen (1972), en su modelo del "cesto de basura," en esta perspectiva todo está abierto. No hay decisiones vinculadas de manera rígida con ciertas premisas, sino que el gobierno local debe resolver todo un complejo de factores y motivaciones para buscar la mejor forma de realizar sus fines. Desde esta perspectiva el sistema organizado estatal es un conjunto de actores y acciones que buscan solucionar problemas. La forma selectiva en que un gobierno local define sus límites es producto de esa selección de problemas, para los que los actores del sistema tienen soluciones contingentes y dinámicas. De la selección de necesidades potenciales, de las que se tienen conocimientos y recursos con los que se pueden diseñar estrategias de acción. Es decir, la selección de la estrategia de cooperación entre actores es la manera en que el gobierno local se diferencia del entorno, configurando una red de acciones internas apoyadas en decisiones de sus actores.

Este planteamiento del gobierno local permite resaltar la importancia de las relaciones sistema organizado y entorno, como una forma de observar eventos y procesos sociales concretos. En este sentido, el gobierno local puede mantenerse, no sólo por su capacidad de adaptación, sino también de que el mismo entorno sea articulado de forma adecuada, es decir, que exista una articulación de actores que colaboren con el sistema, aunque esta cooperación sea de carácter temporal. Esta visión de las relaciones entre entorno social y 
gobierno local, a diferencia de lo planteado por Max Weber (1967) al relacionar todo lo organizado a la existencia de la racionalidad, no supone que un sistema organizado sea simplemente por ello racional, sino que sus objetivos, bases ideológicas o criterios de acción son todo menos racionales. Cuando el entorno es heterogéneo, se requiere una amplia y diversificada red de sistemas organizados que le haga frente a su compleja problemática, en esta situación surgen las redes de acción colectiva o para el caso de los gobiernos locales, redes de política pública. Este colectivo organizado es regulado de diversas maneras según sea el asunto al que hace frente. Este planteamiento se hace en el siguiente apartado.

\section{2) Hacia una reconceptualización del gobierno abierto local}

La descentralización, la pluralidad y alternancia política y la existencia de una sociedad conflictiva han incrementado la complejidad y la incertidumbre en la gestión de las organizaciones públicas locales (Ibarra: 2010), por lo que el principal reto de los gobiernos estatales es crear las condiciones institucionales para la gobernabilidad, para lo cual, en contexto de gobierno dividido, necesitan nuevos mecanismos que regulen su funcionamiento. En este sentido, la gobernabilidad debe ser entendida como la capacidad de ejercer el poder, adoptando una estructura más participativa, diferente al modelo jerárquico, en donde, el gobierno local ejercía un poder centralizado sobre los diversos actores de la sociedad. En la nueva gobernabilidad ninguno de los actores involucrados en los asuntos públicos está por encima de los demás, por lo que las formas organizativas de los gobiernos estatales deberán caracterizarse por la existencia de una estructura horizontal, con actores más autónomos y la adopción de redes organizativas para el diseño y operación de sus políticas públicas.

El funcionamiento de los gobiernos locales con base en una reconfiguración institucional requiere de actores autónomos dispuestos a llegar a acuerdos, que aunque provisionales, permitan el diseño y operación de las políticas públicas. Esto necesita de la adopción de una forma organizacional basada en redes, lo cual implica una serie de condiciones estructurales e institucionales previas. La condición más general para poder desarrollarla es que el poder debe estar disperso en la sociedad, pero no de manera fragmentada e ineficiente. Las autoridades políticas tienen que ser fuertes, pero no omnipotentes. Tienen que haber sido legitimadas democráticamente, de modo que se pueda estimar que los representantes electos reflejan los intereses de todos los grupos sociales. En otras palabras, las autoridades políticas deben poder ser aceptadas, de modo general, como interlocutores entre el Estado y la Sociedad. Es necesario, también, que las autoridades políticas manejen recursos suficientes como para llevar a cabo las decisiones que se tomen, las cuales tienen que ser lo suficientemente 
diversificadas como para responder a las diferentes necesidades de la sociedad.

El funcionamiento gubernamental estatal con base en organizaciones en interacción también requiere de una sociedad civil fuerte, funcionalmente diferenciada e informada. La sociedad local es una comunidad de ciudadanos, de individuos que disfrutan de igualdad ante la ley, así como de un conjunto de derechos fundamentales, y cuya libertad de perseguir sus metas sólo está restringida por el mismo derecho de los demás. Una sociedad no puede existir donde hay una gran desigualdad social, es incompatible con una estructura gubernamental horizontal. Para que exista un funcionamiento gubernamental en red, la sociedad tiene que estar diferenciada funcionalmente en subsistemas, en donde organizaciones específicas lleven a cabo funciones especializadas. Tiene que haber actores organizados que representen los diferentes intereses sociales.

Estas organizaciones deben gozar de una relativa autonomía, esto es, que no deben ser controladas políticamente, como ocurrió en el Estado autoritario, que caracterizó durante mucho tiempo el viejo modelo de Gestión, ahora arcaico a las nuevas exigencias del entorno. En especial, las organizaciones de intereses sociales deben ser suficientemente autónomas y hábiles como para negociar con intereses opuestos y con las dependencias del gobierno estatal. Pero también, entre los diferentes grupos sociales y organizaciones tiene que existir por lo menos, un mínimo sentido de identificación y de responsabilidad con la totalidad, es decir, debe existir una identidad común. Para que haya gobernabilidad los actores involucrados tienen que ser cada uno eficiente en su área de acción, sin que ninguno domine a los demás, y deben cooperar en el diseño, formulación e implantación de políticas públicas, en vez de limitarse a disputar los recursos de la acción pública.

Para lograr las condiciones institucionales y estructurales necesarias para el funcionamiento en red, el proceso de cambio debe iniciar a nivel del gobierno, aunque se piense esta nueva forma organizativa significa una pérdida de la capacidad de dar direccionalidad a las políticas públicas. Sin embargo, de lo que se trata en realidad no es tanto la pérdida de control, sino más bien de que éste cambie de forma. Los actores gubernamentales que participan en las redes de políticas juegan un papel especial y privilegiado, puesto que tienen medios fundamentales de intervención. Cuando se delega la toma de decisiones a actores sociales, los gubernamentales conservan el derecho de ratificación legal, así como el derecho de intervención mediante acciones de supervisión y control, en lo que en la teoría de la gobernanza se conoce como la función de direccionalidad.

La formulación de políticas públicas locales en estructuras organizacionales en red no es algo armonioso. Si en la negociación entran en 
juego intereses en conflicto, cuando se busca el acuerdo para una decisión conjunta, el resultado es una cooperación temporal. Una cooperación de este tipo corre el riesgo de acabar en un completo bloqueo o de producir soluciones temporales. Por ello, el problema básico es evitar los estancamientos y asegurar la solución efectiva de los problemas públicos.

El modelo de gestión del gobierno abierto plantea la necesidad de transformar a los gobiernos estatales, superando la estrategia de reforma administrativa, que hasta ahora ha predominado. La clave está en poner como prioridad el cambiar los mecanismos de regulación que determinan la estructura y funcionamiento de la administración pública. La tarea es encontrar las reglas y normas más pertinentes que permitan la pluralidad, transparencia, eficiencia y rendición de cuentas. Por ello, es necesario transformar la "capacidad institucional", entendida como la capacidad estatal para establecer y garantizar el conjunto de normas en que se apoya la acción colectiva. En este sentido el punto clave es el cambio institucional es cuando se da una transformación en el comportamiento de los actores. Por ello, es importante analizar a partir de experiencias concretas de acción gubernamental la naturaleza compleja de este planteamiento.

\section{3) Gobierno Abierto desde la perspectiva de un Modelo de Gestión}

En las últimas décadas, México ha sido testigo de una transformación en las formas de organización y acción gubernamental. El marco conceptual que subyace a la mayoría de estos cambios está formado por un conjunto de valores, principios y técnicas que surgen en las llamadas ciencias de gestión. Al concepto de gestión se añade el adjetivo público, a partir de lo cual se ha convertido en el punto de referencia de los programas de modernización administrativa. Con ello se da el surgimiento de la llamada "Nueva Gestión Pública", que constituye una de las tendencias internacionales más notables en las organizaciones públicas, convirtiéndose en la principal estrategia de reforma burocrática en numerosos países, desde finales de los años setenta (Hood, 1991).

En el caso del sector público, el concepto "gestión” refleja las circunstancias en las que se encuentran las organizaciones para adaptarse a la complejidad que le plantea la sociedad. El nuevo contexto del sector público reconoce nuevas circunstancias económicas, políticas y sociales que ponen de manifiesto las limitaciones de la perspectiva burocrática. Por ello se ha encontrado en la "Gestión Pública" la promesa de superar la presión económica sobre recursos escasos, atender las demandas insatisfechas de los usuarios de los servicios públicos y racionalizar las decisiones políticas de gobiernos de cualquier partido político. En este panorama las aportaciones de la "gestión" a la hora de dar respuesta a los retos de la modernización de las 
organizaciones públicas, que para efectos de esta obra específicamente nos referiremos a los gobiernos estatales de México.

Para lograr estos objetivos, es necesario, primero hacer una breve reconstrucción sobre la tradición de las ciencias de gestión en las organizaciones públicas, poniendo en énfasis en el escepticismo que generó esta nueva forma de acción estatal. Así por ejemplo, durante la década de los 70 del siglo XX, la gestión pública se presentó como un conjunto de doctrinas de aplicación general y universal, que se caracterizaba por su neutralidad política. Esto significaba, por una parte, que sus principios y técnicas se presentaron como una respuesta válida para los problemas de las organizaciones públicas en multitud de países y en los más variados sectores.

Para principios del siglo XXI se ha respetado la expresión original empleada por los autores de la gestión pública, sobre todo en lo que se refiere a su neutralidad política, a poner en práctica voluntades de ideologías contrapuestas, ofreciéndose como un marco apolítico, bajo el que podían convivir políticas neoliberales y políticas del Estado del bienestar. En las sociedades contemporáneas el proceso político se caracteriza por el pluralismo, fragmentación y diversidad tanto en objetivos como en acciones (Metcalfe, 1987). Esto significa, que la incorporación al sector público de técnicas de gestión de organizaciones privadas no pueda concebirse sólo como una elección de un conjunto de técnicas, sino que es todo un proceso que desea crear las condiciones jurídicas, estructuras y de comportamiento, que sean la condición para que obtengan los resultados esperados. Hay que tomar en cuenta que la especificidad de los problemas públicos locales tiene elementos que no están presentes en los problemas de las organizaciones privadas, como es el caso de la politización, la ambigüedad de los objetivos y un gran número de actores involucrados.

\section{4) Metodología: Valores dominantes del Gobierno Abierto}

Para evaluar el alcance ideológico de los programas de la apertura del gobierno local, es necesario profundizar no sólo en el marco de responsabilidad en el que se expresa la legitimidad de las funciones directivas de los funcionarios públicos, sino también considerar los valores de racionalidad económica, eficiencia y eficacia de la acción pública. La identificación de los valores que subyacen a la concepción de la gestión en el gobierno abierto difícilmente puede ser objeto de una interpretación unitaria. Por una parte, es normal que el sesgo ideológico de los programas se perciba en los valores dominantes, pero, por otra parte, la modernización administrativa ha experimentado una evolución paulatina en sus valores de referencia, superando la simplicidad que poco a poco se ha ido adecuando para lograr cambios profundos y duraderos. 
Pero el uso de estos conceptos, aparentemente sencillos, en el contexto de la Administración Pública Local, presentó grandes dificultades. De un lado, la decisión sobre la opción a seguir para incrementar la eficiencia no puede basarse en criterios puramente técnicos, sino que ésta puede verse afectada por factores políticos. Por otro lado, hay que considerar la dificultad, no de cuantificar, sino de definir los resultados de numerosas actividades públicas donde predominan criterios políticos y por lo tanto difíciles de medir. El concepto de eficiencia presenta, además, diversas interpretaciones, que permiten concluir el carácter reduccionista de sus primeras iniciativas de modernización administrativa. Metcalfe y Richards (1987) distinguen entre la eficiencia técnica, que mide recursos y resultados obtenidos, así como de eficiencia económica. Esta segunda, puede separarse en eficiencia distributiva, óptima distribución de los recursos y la eficiencia dirigida a optimizar la productividad de los recursos empleados en una actividad concreta. El enfoque de eficiencia que asumen los programas de modernización es precisamente ésta última, que se traduce en la búsqueda de reducción de costos. La eficiencia, así definida, resulta un valor problemático, sobre todo cuando no existe una medida previa.

La definición tradicional de eficacia responde al grado en el que se alcanzan los objetivos previamente definidos. Sin embargo, es frecuente que en las organizaciones públicas locales los objetivos no estén definidos o sean múltiples y contradictorios o estén sujetos a cambios constantes en el tiempo. Por ello, el pensamiento organizativo ha recurrido a reformular el concepto de eficacia, dándole las condiciones de flexibilidad, por lo que una noción de eficiencia basada en la homogeneidad y la estandarización se enfrente con demandas de diversidad y duplicidad; por ello, Metcalfe y Richards (1987), hacen la distinción entre el concepto de "Eficiencia Operativa”, que está presente en los programas de modernización y que es coherente con la presencia de objetivos precisos y duraderos, y un concepto de "Eficiencia Adaptativa” que se presenta en las circunstancias inversas. Las circunstancias económicas, políticas y sociales demandan al sector público una mayor flexibilidad y capacidad de adaptación frente a cambios previsibles e imprevisibles en su entorno. También puede advertirse en los últimos años una evolución de gobierno abierto hacia un repertorio más amplio de valores, que tienden a desplazar a la eficiencia del centro de atención y ubicarse en la participación social como un instrumento no sólo de eficiencia sino también de legitimidad política.

De acuerdo a Cabrero y Nava “La participación social desde la perspectiva de un gobierno local puede tener al menos cuatro funciones y de ellas se derivan los mecanismos a instrumentar. La primera se refiere a emitir opiniones al respecto a las preferencias o niveles de satisfacción sobre 
programas o políticas propuestos o ejecutados por el gobierno local (función consultiva), todo ello mediante sondeos de opinión, a través de referéndum, de comités o consejos diseñados para tal fin. La segunda se refiere a colaborar o cooperar en la realización de alguna obra pública o en la prestación de algún servicio (función cooperativa), para esto la constitución de comités, asociaciones de ciudadanos o integración de organismos no gubernamentales, parecerían los mecanismos más usuales. La tercera función se refiere a contribuir en el proceso de toma de decisiones no sólo emitiendo opiniones, sino orientando las acciones del gobierno mediante un poder de decisión (función decisoria), para llevara acabo esta decisión es necesario integrar consejos ciudadanos o agentes sociales, con el fin sólo de contribuir al proceso de deliberación, sino incluso con la autoridad para decidir. Por último, aquella que se refiere a la evaluación y seguimiento de las acciones de gobierno local (función de evaluación), en este sentido la conformación de comités ad hoc a los programas o políticas de que se trate, tanto conformados con ciudadanos como con expertos, serían los mecanismos más comunes. Sin embargo, no todas las funciones que puede abarcar la participación social deben de ser tomadas en cuenta en todo tipo acción de un gobierno, ciertos programas por su naturaleza son más adecuados para una cierta modalidad de participación; además al momento por el que atraviesa la relación entre gobernantes y gobernados, así como el tipo de debate, consensos o conflictos que genere un programa, también serán determinantes para promover la participación de una manera o otra. A partir de estas ideas se podrán considerar dos variables como fundamentales para el análisis de los mecanismos de participación ciudadana a promover: por una parte el nivel de complejidad técnica y de implicaciones de ese tipo que conviene el programa o política en cuestión, y por la otra el nivel de propensión al consenso o al conflicto que el programa genera en la población” (Cabrero y Nava: 1999, p. 85).

Asimismo, propone la siguiente representación visual, que permite tener un referente de modelo de análisis para orientar una reflexión y análisis para conformar estrategias para la toma de decisiones gubernamental e integración de los actores sociales, en un contexto de gobierno abierto. 
Cuadro No.1: Modelo de análisis sobre mecanismos de participación social”

Propenso al consenso o al conflicto en grupos participante.

\section{Conflicto}

Conflicto
Propenso al consenso o al conflicto en
grupos participante.

\section{Consenso}

\begin{tabular}{|c|c|}
\hline $\begin{array}{l}\text { Mecanismos consultivos } \\
\text { Mecanismos } \\
\text { cooperativos }\end{array}$ & $\begin{array}{l}\text { Mecanismos } \\
\text { consultivos }\end{array}$ \\
\hline $\begin{array}{l}\text { Mecanismos consultivos } \\
\text { Mecanismos consultivos } \\
\text { Mecanismos de decisión } \\
\text { Mecanismos de } \\
\text { evaluación }\end{array}$ & 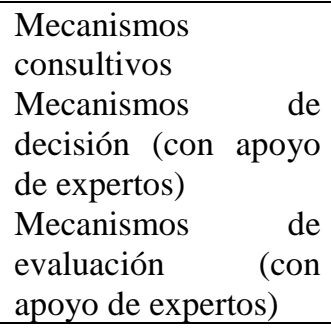 \\
\hline
\end{tabular}

Baja

Alta

Fuente: Elaboración propia en base a Cabrero, E. y Nava, G.; (1999).

Cabe hacer mención, que los aspectos relacionados con las rutas criticas para construir y fomentar una mayor cultura en la participación ciudadana, es decir de construir una gobernabilidad en el sentido amplio que significa este concepto, requiere no sólo de una capacidad de gestión de los conflictos, depende también de una capacidad de gestión de los servicios, de una capacidad de gestión de los bienes públicos, y sobre todo de la capacidad de integrar todo esto en programas y políticas concretas. Así pues, en los últimos años, el marketing estratégico, la calidad total y la gestión por resultados han desplazado al taylorismo como la principal fuente de inspiración de la reforma del gobierno local, tomando prestados sus valores de adaptabilidad, innovación, representación y participación de otras corrientes conceptuales de las teorías de la organización. Entre los valores que caracterizan al nuevo paradigma destacan la calidad de servicio frente a la eficiencia, la productividad frente a la administración, el énfasis en la creación de valor frente a la justificación de los costos y la obtención de adhesión a las normas frente al mero control de su aplicación.

\section{5) Resultados: Modelo de gestión del Gobierno Abierto como paradigma explicativo de la gobernabilidad}

\section{1) Principios para la implementación del gobierno abierto}

Las transformaciones recientes de la Administración Pública Local se orientan hacia una mayor flexibilidad, diversidad estructural y descentralización. Con la apertura a las fuerzas del mercado, las configuraciones organizativas para la prestación de los servicios públicos están destinadas a ser más numerosas y complejas, haciendo más diversos los espacios interorganizativos en los que deben cumplirse los objetivos gubernamentales. Las funciones del gobierno local se han complejizado, transformando sus características, retrocediendo en sus funciones de 
intervención directa y ampliando sus responsabilidades en la satisfacción indirecta de los problemas públicos. A la vez, la principal preocupación central de la administración pública se traslada de la búsqueda de la eficacia y la eficiencia de organizaciones, a una cogestión de diversas organizaciones tanto públicas como privadas.

El gobierno abierto se inscribe en una lógica de macro - proceso organizativos, diferentes a los de las organizaciones principalmente en lo que respecta al contexto y a la ambigüedad en los objetivos. La Administración Pública funciona en redes de organizaciones, las cuales proporcionan el marco de valores en los que operan las agencias gubernamentales a nivel micro.

En este contexto las Ciencias de Gestión se convierten en un paradigma que ofrece un marco de interpretación a los problemas del funcionamiento interno de las organizaciones gubernamentales en un contexto incierto, dinámico y conflictivo y por lo tanto permite inducir la capacidad de gobernar de los Estados. En donde los planteamientos hechos con base en los principios de eficacia y eficiencia, ligadas a un modelo de pensamiento individualista y racional, dejan de ser apropiadas, debiendo reinterpretarse con base en valores colectivos de participación, representatividad e igualdad y considerando la diversidad de las formas y estrategias organizativas de los gobiernos locales. El modelo de Gestión de Gobierno Abierto, desde la perspectiva de las Ciencias de Gestión es una aproximación a nivel micro del comportamiento de la acción pública. Por esta razón, es un concepto que es compatible con el de gobernabilidad, entendida como la evolución de la administración pública hacia la superación de la dicotomía público-privada, a una dinámica de interacción constante entre diversas organizaciones tanto sociales, políticas, económicas y gubernamentales.

De acuerdo con el manual de Gobierno Abierto, publicado por la Comisión Económica para América Latina y el Caribe (CEPAL), ha conceptualizado que, se ha mimetizado gradualmente como una alternativa de innovación pública y adaptada para fortalecer las capacidades institucionales de la administración pública local. Todo ello, ha quedado de manifiesto en que cuando se habla de gobierno abierto, se hace referencia al menos a los siguientes dos ejes: a) Promover una cultura de transparencia e institucionalización de acciones que faciliten el acceso a la información y su potencial reutilización (con fines de control social o político, de generar valor público, cívico o económico), al mismo tiempo que se facilitan los espacios de fortalecer la integridad pública y la rendición de cuentas; b) Fortalecer los espacios de participación ciudadana en los asuntos públicos y en la toma de decisiones que les atañen, promoviendo además la colaboración en la búsqueda e implementación de soluciones en un esquema de mayor responsabilidad compartida que pueda aprovechar las capacidades distribuidas y la inteligencia colectiva de los actores sociales. 
Dicho modelo tiene como objetivo:

- Mejorar los niveles de transparencia y acceso a la información mediante la apertura de datos públicos (para ejercer control social sobre los gobiernos y facilitar la rendición de cuentas) y la reutilización de la información del sector público (para promover la innovación y el desarrollo económico).

- Facilitar la participación ciudadana en el diseño e implementación de las políticas públicas (e incidir en la toma de decisiones).

- Favorecer la generación de espacios de colaboración e innovación entre los diversos actores, particularmente entre las administraciones públicas, la sociedad civil y el sector privado, para codiseñar y/o coproducir valor público, social y cívico. (Naser y Ramírez, 2014).

- Innovación en materia de gobiernos locales es readecuar las estructuras de administración, los mecanismos de interacción con la ciudadanía, y el marco institucional de gobierno a un nuevo escenario, de tal forma que los resultados sean mejor tanto en la calidad de las acciones y servicios del gobierno, como en la gobernabilidad democrática y en la confianza de los ciudadanos. (Cabrero, 2002).

Dicha evolución se puede ver reflejada en acciones concretas, tales como se presentan en la siguiente figura.

Figura 1: Los Principios del Gobierno Abierto en acción

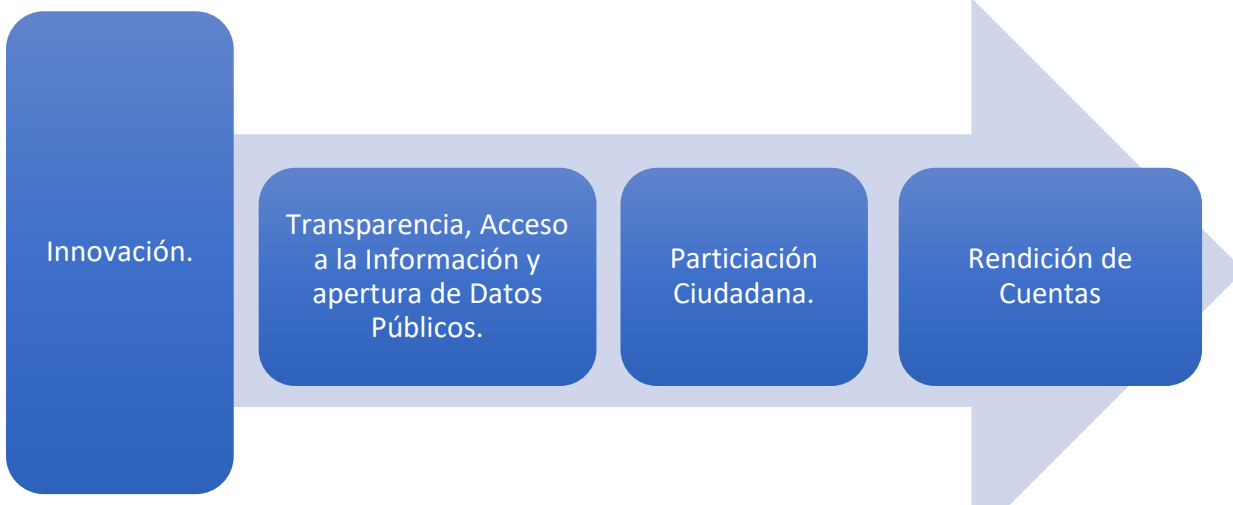

Fuente: Elaboración propia con base en Ramírez, Alujas y Dassen, 2012.

La propuesta de este modelo de Gestión del Gobierno Abierto, esta representada en el (figura No.1), en que se visualizan cuatro principios, aunque el del concepto de innovación, lo pudiésemos considerar como transversal a dichas acciones. En el caso de la innovación se puede materializar para mejorar el impacto final de la acción del gobierno local. Las restructuraciones 
internas, la adopción de nuevos sistemas de gestión, el diseño de nuevos mecanismos de interacción con la ciudadanía, son solo medios para el fin último: Mayor bienestar social y un clima democrático y abierto entre gobernantes y gobernados.

En el caso del principio de la transparencia, acceso a la información y apertura datos públicos, que se pueden traducir en acciones concretas, tales como: portales de transparencia; mecanismos de rendición de cuentas; plataformas de acceso a datos públicos abiertos; normativas de reutilización de la información pública. Así mismo, en el que se refiere al de participación ciudadana, se visualizan con acciones como el diseño de plataformas de consulta y un rol activo en el diseño y formulación de políticas públicas, mecanismos de escucha activa y canales de doble vía (Gov 2.0); promoción de espacios para iniciativas ciudadanas, servicios digitales y/o servicios en línea. Por último, el principio de colaboración se refiere a plataformas de trabajo colaborativo, iniciativas de codiseño, cocreación, y coproducción de servicios de valor (público, económico, social y cívico), inteligencia colectiva aplicada a la gestión público - privada / público - social.

Así pues, ante todos estos referentes conceptuales propuestos en el modelo, se pueden materializar con la siguiente representación esquemática del "Modelo de Gestión de Gobierno Local Abierto". (Ver figura No. 2).

Figura 2: Modelo de Gestión de Gobierno Abierto Local

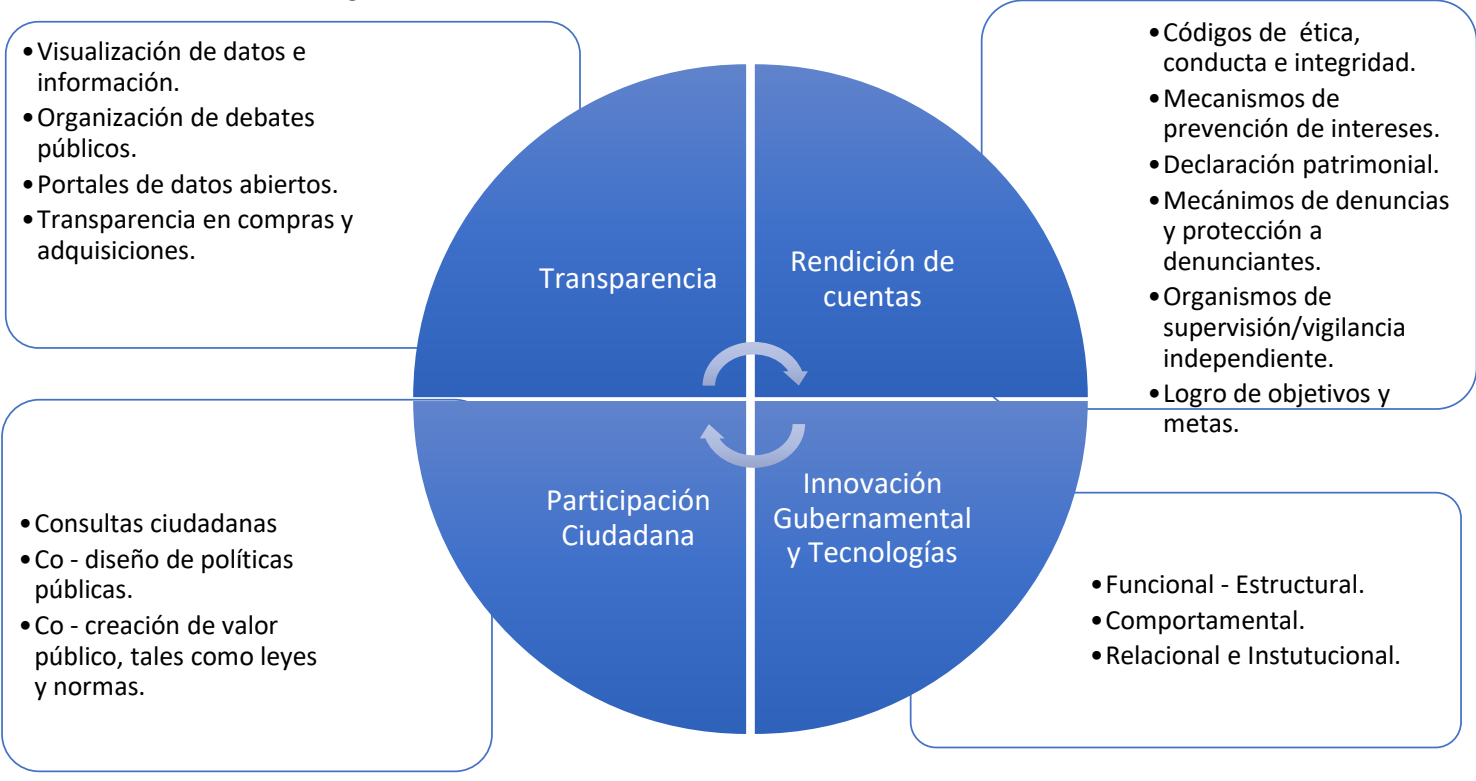

Fuente: Elaboración propia en base en Ramírez - Alujas y Dassen, 2012.

Cabe destacar que el apartado de innovación y tecnología requiere de una explicación más particular. En ella, se refiere a los tipos de innovación, incorporando la tecnología, y puede dividirse en tres grandes apartados: La 
primera se refiere a la Estructural - Funcional, lo que significa hacer nuevas cosas de una nueva manera, nueva combinación de recursos, nuevas formas organizativas; El segundo, denominado Comportamental, promueve el cambio en nuevas actitudes, valores y arreglos intraorganizacionales, así como una transformación en el proceso de toma de decisiones y por último el Relacional e Institucional que integra nuevas formas de relación organización - sociedad, nuevos arreglos institucionales, entendiéndose como "Institución", aquella organización que prevalece a lo largo del tiempo sin importar los actores que las dirigen o componen y que son aceptadas por la mayoría de la sociedad. De acuerdo a la Alianza para el Gobierno Abierto, se refiere a planes de acción, que además, deberán reflejar y estar guiados por los cuatro principios centrales de Gobierno Abierto, tal y como se representa en el esquema anterior, siendo los siguientes: Transparencia, Participación Ciudadana, Rendición de Cuentas e Innovación y Tecnología.

Tabla 1: Ejes rectores del Modelo de Gobierno Abierto Local

\begin{tabular}{|l|l|}
\hline \multicolumn{1}{|c|}{ Principio } & \multicolumn{1}{c|}{ Conceptualización } \\
\hline 1. Transparencia & $\begin{array}{l}\text { Se refiere a la información sobre las decisiones y el quehacer } \\
\text { gubernamental deben ser abiertos, completos, oportunos gratuitos y } \\
\text { de fácil acceso para el público. Ello supone que los datos públicos } \\
\text { deben de cumplir con parámetros y estándares comúnmente } \\
\text { reconocidos, tales como estar disponibles en formatos, susceptibles } \\
\text { de ser procesados, que puedan ser accesibles a través de herramientas } \\
\text { tecnológicas y de comunicación. }\end{array}$ \\
\hline $\begin{array}{l}\text { 2. Participación } \\
\text { ciudadana }\end{array}$ & $\begin{array}{l}\text { Los gobiernos deberán de buscar que la ciudadanía se interese e } \\
\text { involucre en el debate público, proveyendo los canales apropiados } \\
\text { (aportando información y espacios de consulta) y mediante } \\
\text { contribuciones que conduzcan a una gobernanza más efectiva, } \\
\text { innovadora, responsable y que atienda las necesidades de la sociedad. }\end{array}$ \\
\hline $\begin{array}{l}\text { 3. Rendición de } \\
\text { cuentas }\end{array}$ & $\begin{array}{l}\text { Supone la existencia de normativas, procedimientos y mecanismos } \\
\text { para que los servidores públicos justifiquen sus acciones, reaccionen } \\
\text { a requerimientos y/o criticas que se plantean, y asuman la } \\
\text { responsabilidad por sus actos u omisiones, o cuando se apartan de la } \\
\text { normativa o de los compromisos asumidos. }\end{array}$ \\
\hline $\begin{array}{l}\text { 4. Innovación y } \\
\text { Tecnología }\end{array}$ & $\begin{array}{l}\text { Prever a la ciudadanía un acceso más abierto a las nuevas tecnologías, } \\
\text { y al relevante rol que ellas tienen en la innovación, así como la } \\
\text { importancia de éstas para aumentar la capacidad de los ciudadanos en } \\
\text { el uso que de ellas hacen. }\end{array}$ \\
\hline
\end{tabular}

Fuente: Elaboración propia en base al documento denominado: "La Alianza de Gobierno Abierto en México, una visión de la sociedad civil” en (Barrera, 2015).

Para concluir y cerrar este apartado, se refirió a una visión global del estado de arte sobre el gobierno abierto a la fecha, que complemente lo desarrollado previamente y sea de utilidad como documento de trabajo que permitirá reafirmar los principios, componentes y herramientas para implementar y desarrollar una representación abstracta teórico - conceptual de la interacción entre gobierno y ciudadanía para la construcción de consensos 
y procesos decisorios de la gestión pública estatal, en la solución de problemas planteados que inciden en el bienestar de la sociedad, de tal manera que permita dicho modelo analizar, describir, explicar y predecir estos procesos.

\section{2) Propuesta metodológica para analizar el Gobierno Abierto}

En este apartado se hace una propuesta metodológica que permitirá analizar, describir y explicar dichos procesos, en las cuatro dimensiones, que de acuerdo con la conceptualización del Modelo de Gestión de Gobierno Abierto en el apartado que le antecede, la cual incorpora principios, políticas y/o acciones de transparencia, acceso a la información, rendición de cuentas, participación ciudadana y cocreación gubernamental apoyadas en las TIC’s. Para ello se tomó como referente el enfoque SMART. ${ }^{1}$ La manera en que el usuario que utilice este documento de trabajo deberá de evidenciar documentalmente los compromisos y esfuerzos que reflejen conforme la evolución de los cambios y transformaciones de manera innovadora que en materia de gobierno abierto puedan formular.

Dicho enfoque propuesto en el documento denominado "Guía de Gobierno Abierto"(2012), menciona que de acuerdo a las características para lograr integrar el modelo de gobierno abierto se requieren cinco aspectos a considerar, tales como: 1. Especificidad en el compromiso, es decir, resumir las actividades concretas que se llevarán a cabo para lograr el cumplir con los compromisos; 2. Medible, con puntos de referencia - métrica por acción que se pueda medir, son necesarios para el seguimiento del proceso y se incorporarán al proceso de evaluación a través de mecanismos de revisión independiente; 3. Realizable, deberá de incluirse una breve explicación de cómo se alcanzarán los resultados y las metas; 4. Relevantes, en relación a la visión del gobierno abierto y por último; 5. El tiempo, es decir los compromisos no deberán de tener ambigüedad en los plazos de inicio y término. Tal y como puede observar a continuación. (Ver figura No. 3)

${ }^{1}$ Este enfoque por sus siglas en inglés se refiere a (Specific, Measurable, Actionable, Relevant, Tome - bound), mismos que son presentados del documento "Open Goverment Partnership Orientation Packet” en: “Alianza para el Gobierno Abierto”, (2014). 
Figura 3: Metodología del Modelo de Gobierno Abierto Local

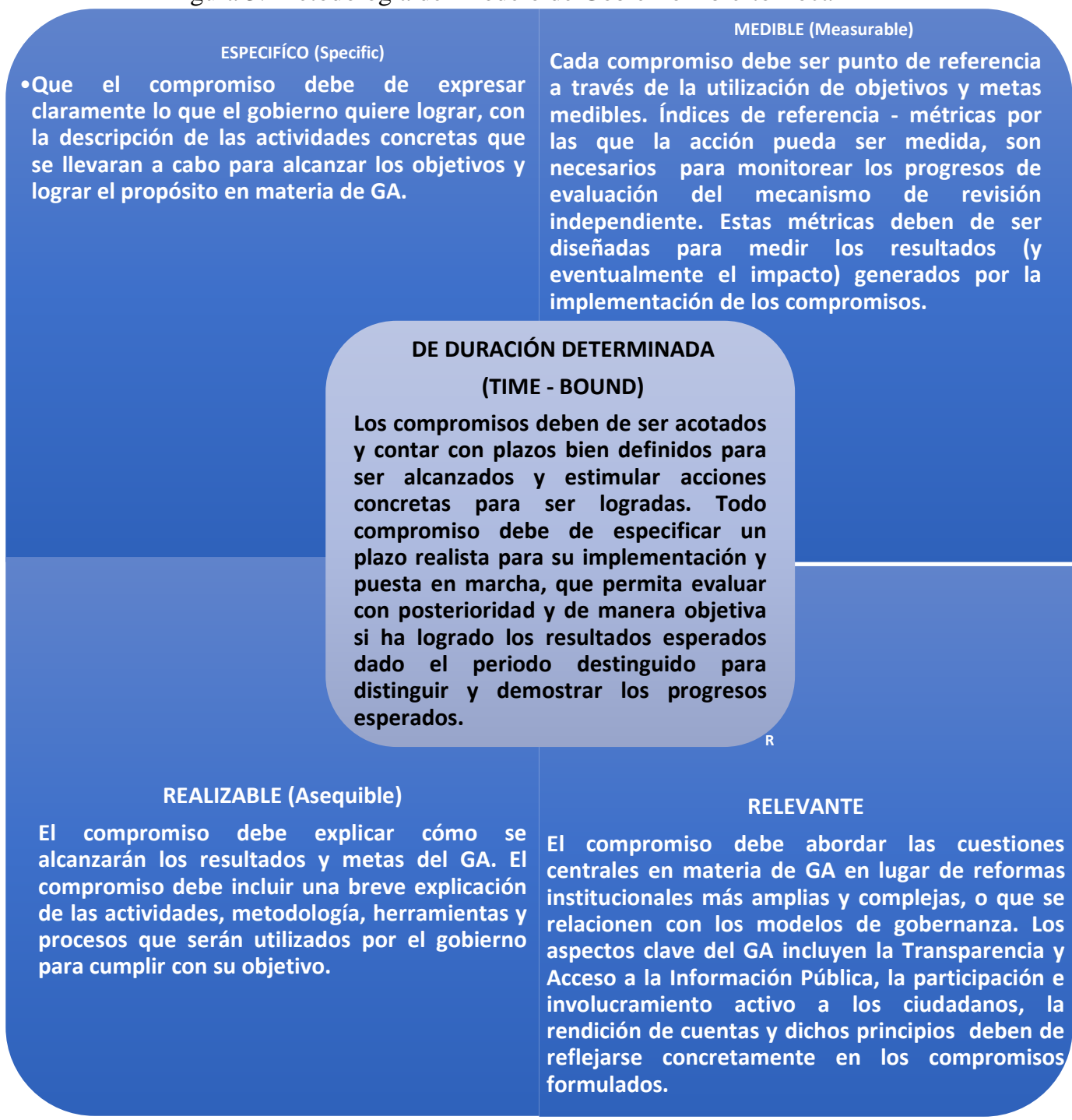

Fuente: Elaboración propia en base a las ideas contenidas en el documento “Open Goverment Partnership Orientation Packet” (2012).

Para finalizar este apartado que incluye una propuesta base y concreta para documentar el Gobierno Abierto Local, deberá de ser consciente de sus alcances y limitaciones en materia de gobierno abierto, mismas que dependerán de las circunstancias de cada una de las instituciones, sus fortalezas, debilidades, oportunidades, amenazas expresadas al interior y exterior de éstas y que pueden ser aplicable a diversas realidades, y reconociendo algunos estándares mínimos para su cumplimiento. 


\section{Conclusion}

Como lo acabamos de ver, los gobiernos abiertos son espacios donde se perciben de mejor manera las transformaciones globales, pero a la vez son actores activos de la transformación económica, política y social. Los cambios más relevantes que han sucedido en las últimas décadas-cambio demográfico, globalización de la información y el capital, desarrollo tecnológico- han generado un impacto en la forma que los gobiernos se estructuran y ejercen su capacidad de gobernar. Lejos de dejar a los gobiernos locales al margen de la gobernabilidad, éstos se han convertido en espacios de intercambio de información donde el contacto interpersonal ha mantenido su importancia. El nuevo rol de los gobiernos abiertos ha generado nuevos procesos de gobierno. En los últimos años, este tipo de gobiernos han modificado su estructura, modernizado sus procesos de trabajo, y complejizado su proceso de toma de decisiones. De ser sólo prestadores de servicios públicos han pasado a ser impulsores del desarrollo local mediante políticas públicas de carácter endógeno. Para adoptar esta nueva forma de gobierno ha incorporado actores de la sociedad, de este proceso proviene el concepto de gobernanza, que sugiere la articulación cotidiana del gobierno y la sociedad.

Estos nuevos procesos de gobierno pueden interpretarse como un síntoma de un fenómeno social más amplio, producto de cambios no sólo en lo económico, sino también en lo político y a la persistencia de los grandes déficits sociales como la pobreza, la marginación y la exclusión social. En este contexto, el modelo de Gobierno Abierto, en donde la sociedad recupera espacios en la gestión de los asuntos públicos. El Gobierno Abierto en un territorio especifico, desde un enfoque local, aparece vinculado a las interacciones que se definen y se conforman con base en el consenso y el aprendizaje colectivo de los actores con sus distintas lógicas acción y posibles desacuerdos.

La capacidad de integrar y dar forma a los múltiples intereses locales, representarlos hacia el exterior y generar estrategias más o menos unificadas a lo largo del tempo aparece como la principal ventaja de este modelo de gestión. El gobierno abierto conforma así un tipo particular de gobernar cuyo eje está centrado en la construcción de nuevas modalidades de intervención y articulación más participativas y cooperativas entre los distintos actores e instancias institucionales de las sociedades locales.

En la mayor parte de los gobiernos estatales en México se ha vivido un proceso de transformación en la forma de hacer frente a los problemas públicos. Este proceso se ha caracterizado por la transición de un modelo centralizador en la hechura de políticas a uno de mayor interacción con la sociedad local. Es decir, este proceso de transformación en la hechura de las políticas ha sido producto, de la transferencia de mayores atribuciones y 
recursos por parte del gobierno central, pero también de una nueva dinámica social, económica y política en los espacios locales.

Por ello, es la innovación de los gobiernos locales la principal fuente explicativa de un nuevo modelo de gestión local y su principal impacto ha sido en el proceso de hechura de las políticas. La innovación se refiere a la capacidad de los gobiernos locales para estructurar de manera diferente los recursos de que dispone para hacer frente a los problemas públicos. Esta innovación tiene dos dimensiones principales, estructura organizacional y comportamental (Cabrero, 2005), con lo cual han buscado incrementar los niveles de eficiencia, eficacia y credibilidad, generando nuevos procesos de interacción en la acción pública, modificando patrones de comportamiento, criterios en la toma de decisiones y rol de los actores de la sociedad.

Sin embargo, en los gobiernos locales de México, estos modelos de gestión de Gobierno Abierto no se encuentran de manera pura, ni generalizada, sino que pueden coexistir en un mismo gobierno local en una misma administración, o en una misma oficina, ya que dependen de los asuntos públicos que se les hace frente. Con ello surge una nueva característica de esta perspectiva, la fragmentación y con ello la flexibilidad en las formas de organización del gobierno local.

Por último, las grandes transformaciones desarrolladas en los últimos años en las formas de gobierno y del proceso de hechura de las políticas públicas plantean la necesidad de enfoques conceptuales explicativos que den cuenta de la articulación y participación de múltiples actores en el proceso de solución de los problemas públicos. El tránsito de un estilo de gobierno centralizado y jerárquico hacia prácticas más pluralistas y cooperativas supone claramente un replanteamiento de los marcos interpretativos del gobierno local, que requiere pasar de un énfasis normativo a uno que ponga énfasis en el actor y sus acciones, donde tiene cabida esta propuesta conceptual de modelo de gestión abierta.

\section{References:}

1. Barrera, L.; (2015); La alianza para el Gobierno Abierto, una visión desde la sociedad civil. México: Creative Commons.

2. Cabrero, E. y Nava, G.; (1999); Gerencia Pública municipal. Conceptos básicos y estudios de caso”. México: Editorial Miguel Ángel Porrúa.

3. Cabrero, E.; (2002); Premio Gobierno y Gestión Local. Innovación en Gobiernos Locales: Un panorama de experiencias municipales en México. México: Centro de Investigación y Docencia Económicas.

4. Cabrero, E.; (2005). Acción pública y desarrollo local. México: Fondo de Cultura Económica. 
5. Cohen, M., March, G. y Olsen. P.; (1972). “A garbage can model of organizatio- nal choice”; Administrative Science Quarterly, 17.

6. Hood, C.; (1991) “A public management for all seasons?”; en Public Adminitration, Vol. 3, No 7, 1993.

7. Ibarra, M.; (2010). Estrategia de política pública en la gestión financiera en los municipios de urbanos de México y su impacto en la gobernabilidad local. México: Editorial Miguel ángel Porrúa.

8. Ibarra, M. Santos, J. (2011). Actores locales y sus acciones para el análisis de la gobernabilidad a nivel municipal”. México: Editorial Universitaria Potosina.

9. Luhmann, N.; (1994); Funktionen und Folgen formaler Organisation. Berlin: Funciones y consecuencias de la organización formal.

10. Mballa, Louis Valentin (2017), Políticas públicas y complejidad: en búsqueda de soluciones a los problemas públicos, México: Miguel Ángel Porrúa.

11. Mballa, L.V. (2018), “La racionalidad y contexto políticoinstitucional en la ideación de las políticas públicas”, Revista de Estudios Políticas, XIV(45), 177-202, México: UNAM.

12. Metcalfe, L. y Richards, E. (1987). Public Management: From Imitation to Innovation. México: Herrero Hermanos.

13. Naser, A. Ramírez, A. (2014). "Plan de Gobierno Abierto. Una hoja de ruta para los gobiernos de la región” CEPAL, Santiago de Chile: Serie Manuales No. 81.

14. Open Goverment Partnership Orientation Packet (2012); Support Unit; Waschington DC.

15. Open Goverment Partnership, Transparency ans Accountability Initiative (2013), Open Goverment Guide. Disponible en : http://www.opengovguide.com/.

16. Pettigrew, A. (1973); The politics of Organizational Decisión Making. London: Tavistock.

17. Ramírez, Alujas, A. y Dassen, N. (2012). “Gobierno Abierto: la ruta hacia una nueva agenda de reforma del Estado y modernización de la Administración Pública en América Latina y el Caribe”; en "Gobierno Abierto y transparencia focalizada. Tendencias y desafíos para América Latina y el Caribe”; Dassen, N. y Cruz, J. (Editores); Banco Inter - Americano de Desarrollo, pp. 41:71. Disponible para descargar en: http://bit.ly/SW y NbR.

18. Weber, M. (1967); El político y el científico. Madrid: Alianza Editorial,

\section{Páginas de Internet:}

19. http:/gobabiertomx.org/alianza-mexico/ Consultado el día 14 de noviembre del 2016. 
20. http://gobabiertomx.org/alianza-internacional/ Consultado el día 14 de noviembre del 2016.

21. http://gobiernoabiertoslp.mx/que-es-el-gobierno-abierto/ Consultado el día 14 de noviembre del 2016.

22. http://www.inicio.ifai.org.mx (Gobierno Abierto, co - creación desde lo local), consultado el: 24 de noviembre de 2016.

23. http://www.opengovpartnership.org/es/node/9256, consultado el 28 de diciembre del 2016.

\section{Documentos consultados:}

24. Acta de Instalación del Secretariado Técnico del Estado de San Luis Potosí para el despliegue del Plan de Acción Local de Gobierno Abierto suscrita el 7 de julio del 2015.

25. Declaración conjunta para la constitución de la Red Potosina de Municipios de Gobierno Abierto, con fecha del 22 de Febrero de 2016 por los 58 Presidentes Municipales del Estado.

26. Periódico Oficial del Gobierno del Estado "Plan de San Luis", Año XCIX, Tomo II del Martes 05 de Julio de 2016. 